\title{
Optimal Convergence Rates for Generalized Alternating Projections
}

\author{
Mattias Fält* and Pontus Giselsson*
}

March 31, 2017

\begin{abstract}
Generalized alternating projections is an algorithm that alternates relaxed projections onto a finite number of sets to find a point in their intersection. We consider the special case of two linear subspaces, for which the algorithm reduces to a matrix iteration. For convergent matrix iterations, the asymptotic rate is linear and decided by the magnitude of the subdominant eigenvalue. In this paper, we show how to select the three algorithm parameters to optimize this magnitude, and hence the asymptotic convergence rate. The obtained rate depends on the Friedrichs angle between the subspaces and is considerably better than known rates for other methods such as alternating projections and Douglas-Rachford splitting. We also present an adaptive scheme that, online, estimates the Friedrichs angle and updates the algorithm parameters based on this estimate. A numerical example is provided that supports our theoretical claims and shows very good performance for the adaptive method.
\end{abstract}

\section{Introduction}

Many methods for finding a point in the intersection of a finite number of sets exist. Notable examples include alternating projections [1, 2], its generalization, generalized alternating projections, that allows for relaxed projections [3, 4, 5], Dykstra's algorithm [6], Douglas-Rachford splitting [7, 8, and its dual algorithm ADMM [9, 10]. Considerable effort has gone into understanding and analyzing performance and convergence rates of these methods. Convex and nonconvex feasibility problems have been analyzed in [11, 12, and convex optimization and monotone inclusion problems in $[8,13,14,15]$.

For feasibility problems with two subspaces, it has been long known that the standard alternating projection method converges linearly with exact rate being the squared Friedrichs angle [16]. The Friedrichs angle is the smallest non-zero principal angle between the subspaces, see [2] for background on principal angles. More recently, it was shown in [17] that the Douglas-Rachford algorithm converges with a rate given by the Friedrichs angle.

These projection based algorithms reduce to matrix iterations when the two sets are subspaces. This was exploited in [18, where sharp convergence rates for matrices

\footnotetext{
${ }^{*}$ Department of Automatic Control, Lund University, Sweden. Email: \{mattiasf,pontusg\}@control.1th.se Both authors are financially supported by the Swedish Foundation for Strategic Research and are members of the LCCC Linneaus Center at Lund University.
} 
are provided. They apply their results to find optimal parameters for the generalized alternating projections method. Two of the parameters are kept fixed and they optimize over the third.

In this paper, we extend the results of [18. We optimize the sharp convergence rate for the generalized alternating projection method over all three algorithm parameters. The obtained optimal rate turns out to be significantly better than the ones considered in [18. The optimal parameters in our setting also depends on the Friedrichs angle. This angle is of course not known a priori. Therefore, we have developed an adaptive scheme that estimates the Friedrichs angle during the course of the iterations. Under easily achievable assumptions on the starting point of the algorithm, we show that it is always a conservative estimate of the true Friedrichs angle. Indeed, in examples we see that the estimated angle approaches the Friedrichs angle.

The intention of this work is not to present a new method for solving linear systems of equations. It is rather a starting point to optimize local linear convergence behavior for the generalized alternating projection method, when solving, e.g., problems with affine and conic constraints. Such feasibility problems can solve essentially any convex optimization problem, by first reformulating the problem as a cone program (which is done in the CVX modeling languages [19, 20, 21]), and then use primal dual embedding, as in 22]. The local convergence analysis of such problems is outside the scope of this paper. Encouraging results have, however, been presented, e.g., in [23] and [24]. They show that the local linear convergence rate for Douglas-Rachford splitting for specific convex optimization problems is exactly the Friedrichs angle, i.e., the same as for subspaces. The results rely on sufficient local smoothness or polyhedral/affine sets and finite identification of active sets or manifolds. The finite identification property implies that locally, the problem reduces essentially to an affine subspace intersection problem.

We verify the theoretical results on numerical examples and demonstrate that the generalized alternating projections with optimal parameters performs significantly better than with previously studied parameters in, e.g., [2, 18. We also observe that the proposed adaptive method performs in line with the method with optimal parameters.

\section{Preliminaries}

Let the inner product and induced norm be denoted by $\langle u, v\rangle$ and $\|v\|:=\sqrt{\langle v, v\rangle}$ for vectors $u, v \in \mathbb{R}^{n}$. Let the set of eigenvalues for a matrix $A \in \mathbb{R}^{n \times n}$ be denoted by $\sigma(A)$, the spectral radius as $\rho(A):=\max \{|\lambda| \mid \lambda \in \sigma(A)\}$ and let $\|A\|$ be the operator norm $\|A\|:=\sup _{x \in \mathbb{R}^{n}:\|x\|=1}\|A x\| . P_{\mathcal{C}}$ is the orthogonal projection onto a closed, convex and nonempty set $\mathcal{C}$, i.e. $P_{\mathcal{C}} x=\operatorname{argmin}_{y \in \mathcal{C}}\{\|x-y\|\}$.

The following definitions and facts follow closely those in the related work [18].

Definition 1 The principal angles $\theta_{k} \in[0, \pi / 2], k=1, \ldots, p$ between two subspaces $\mathcal{U}, \mathcal{V} \in \mathbb{R}^{n}$, where $p=\min (\operatorname{dim} \mathcal{U}, \operatorname{dim} \mathcal{V})$, are recursively defined by

$$
\begin{array}{rll}
\cos \theta_{k}:= & \max _{u_{k} \in \mathcal{U}, v_{k} \in \mathcal{V}}\left\langle u_{k}, v_{k}\right\rangle \\
\text { s.t. } & \left\|u_{k}\right\|=\left\|v_{k}\right\|=1, \\
& \left\langle u_{k}, v_{i}\right\rangle=\left\langle u_{i}, v_{k}\right\rangle=0, \forall i=1, \ldots, k-1 .
\end{array}
$$


Fact 1 [18, Def 3.1, Prop 3.3] The principal angles are unique and satisfy $0 \leq \theta_{1} \leq$ $\theta_{2} \leq \ldots \theta_{p} \leq \pi / 2$. The angle $\theta_{F}:=\theta_{s+1}$, where $s=\operatorname{dim}(\mathcal{V} \cap \mathcal{U})$, is the Friedrichs angle and it is the smallest non-zero principal angle.

Definition $2 A \in \mathbb{R}^{n \times n}$ is linearly convergent to $A^{\infty}$ with linear convergence rate $\mu \in$ $[0,1)$ if there exist $M, N>0$ such that

$$
\left\|A^{k}-A^{\infty}\right\| \leq M \mu^{k} \quad \forall k>N, k \in \mathbb{N} .
$$

Definition 3 [18, Fact 2.3] For $A \in \mathbb{R}^{n \times n}$ we say that $\lambda \in \sigma(A)$ is semisimple if $\operatorname{ker}(A-\lambda I)=\operatorname{ker}(A-\lambda I)^{2}$.

Fact 2 [18, Fact 2.4] For $A \in \mathbb{R}^{n \times n}$, the limit $A^{\infty}:=\lim _{k \rightarrow \infty} A^{k}$ exists if and only if

- $\rho(A)<1$ or

- $\rho(A)=1$ and $\lambda=1$ is semisimple and the only eigenvalue on the unit circle.

Definition 4 [18, Def. 2.10] Let $A \in \mathbb{R}^{n \times n}$ be a (nonexpansive) matrix and define

$$
\gamma(A):=\max \{|\lambda| \mid \lambda \in\{0\} \cup \sigma(A) \backslash\{1\}\} .
$$

Then $\lambda \in \sigma(A)$ is a subdominant eigenvalue if $|\lambda|=\gamma(A)$.

Fact 3 [18, Thm. 2.12] If $A \in \mathbb{R}^{n \times n}$ is convergent to $A^{\infty}$ then

- $A$ is linearly convergent with any rate $\mu \in(\gamma(A), 1)$

- If $A$ is linearly convergent with rate $\mu \in[0,1)$, then $\mu \in[\gamma(A), 1)$.

\section{Optimal parameters for GAP}

Let the relaxed projection onto a set $\mathcal{C}$, with relaxation parameter $\alpha$, be defined as $P_{\mathcal{C}}^{\alpha}:=(1-\alpha) I+\alpha P_{\mathcal{C}}$. The generalized alternating projections (GAP) 25] for two closed, convex and nonempty sets $\mathcal{U}$ and $\mathcal{V}$, with $\mathcal{U} \cap \mathcal{V} \neq \emptyset$, is then defined by the iteration

$$
x^{k+1}:=S x^{k},
$$

where

$$
S=(1-\alpha) I+\alpha P_{\mathcal{U}}^{\alpha_{2}} P_{\mathcal{V}}^{\alpha_{1}}=:(1-\alpha) I+\alpha T .
$$

The operator $S$ is averaged and the iterates converge to the fixed-point set fix $S$ under the following assumption, see e.g. [25] where these results are collected.

Assumption 1 Assume that $\alpha \in(0,1], \alpha_{1}, \alpha_{2} \in(0,2]$ and that either of the following holds

A1. $\alpha_{1}, \alpha_{2} \in(0,2)$

A2. $\alpha \in(0,1)$ with either $\alpha_{1} \neq 2$ or $\alpha_{2} \neq 2$

A3. $\alpha \in(0,1)$ and $\alpha_{1}=\alpha_{2}=2$ 
To study the convergence rate of $S$, and its dependence on the parameters $\alpha_{1}, \alpha_{2}$ and $\alpha$, we need to characterize the eigenvalues of $S$. To this end, we state the following proposition, as found in [18, Prop. 3.4].

Proposition 1 Let $\mathcal{U}$ and $\mathcal{V}$ be affine subspaces in $\mathbb{R}^{n}$ satisfying $p:=\operatorname{dim}(\mathcal{U}), q:=$ $\operatorname{dim}(\mathcal{V})$, where $p \leq q, p+q<n$ and $p, q \geq 1$. Then, the projection matrices $P_{\mathcal{U}}$ and $P \mathcal{V}$ become

$$
\begin{aligned}
& P_{\mathcal{U}}=D\left(\begin{array}{cccc}
I_{p} & 0 & 0 & 0 \\
0 & 0_{p} & 0 & 0 \\
0 & 0 & 0_{q-p} & 0 \\
0 & 0 & 0 & 0_{n-p-q}
\end{array}\right) D^{*}, \\
& P_{\mathcal{V}}=D\left(\begin{array}{cccc}
C^{2} & C S & 0 & 0 \\
C S & S^{2} & 0 & 0 \\
0 & 0 & I_{q-p} & 0 \\
0 & 0 & 0 & 0_{n-p-q}
\end{array}\right) D^{*}
\end{aligned}
$$

and

$$
P_{\mathcal{U}} P_{\mathcal{V}}=D\left(\begin{array}{cccc}
C^{2} & C S & 0 & 0 \\
0 & 0_{p} & 0 & 0 \\
0 & 0 & 0_{q-p} & 0 \\
0 & 0 & 0 & 0_{n-p-q}
\end{array}\right) D^{*}
$$

where $C$ and $S$ are diagonal matrices containing the cosine and sine of the principal angles $\theta_{i}$, i.e.

$$
\begin{aligned}
& S=\operatorname{diag}\left(\sin \theta_{1}, \ldots, \sin \theta_{p}\right), \\
& C=\operatorname{diag}\left(\cos \theta_{1}, \ldots, \cos \theta_{p}\right),
\end{aligned}
$$

and $D \in \mathbb{R}^{n \times n}$ is an orthogonal matrix.

Under the assumptions in Proposition 1, the linear operator $T$, implicitly defined in (2), becomes

$$
\begin{aligned}
T= & P_{\mathcal{U}}^{\alpha_{2}} P_{\mathcal{V}}^{\alpha_{1}}=\left(\left(1-\alpha_{2}\right) I+\alpha_{2} P_{\mathcal{U}}\right)\left(\left(1-\alpha_{1}\right) I+\alpha_{1} P_{\mathcal{V}}\right) \\
= & \left(1-\alpha_{2}\right)\left(1-\alpha_{1}\right) I+\alpha_{2}\left(1-\alpha_{1}\right) P_{\mathcal{U}} \\
& +\alpha_{1}\left(1-\alpha_{2}\right) P_{\mathcal{V}}+\alpha_{1} \alpha_{2} P_{\mathcal{U}} P_{\mathcal{V}} \\
= & D \operatorname{blkdiag}\left(T_{1}, T_{2}, T_{3}\right) D^{*}
\end{aligned}
$$

where

$$
\begin{aligned}
& T_{1}=\left(\begin{array}{cc}
I_{p}-\alpha_{1} S^{2} & \alpha_{1} C S \\
\alpha_{1}\left(1-\alpha_{2}\right) C S & \left(1-\alpha_{2}\right)\left(I_{p}-\alpha_{1} C^{2}\right)
\end{array}\right), \\
& T_{2}=\left(1-\alpha_{2}\right) I_{q-p}, \quad T_{3}=\left(1-\alpha_{2}\right)\left(1-\alpha_{1}\right) I_{n-p-q} .
\end{aligned}
$$

The rows and columns of $T_{1}$ can be reordered so that it is a block-diagonal matrix with blocks

$$
T_{1}^{i}=\left(\begin{array}{cc}
1-\alpha_{1} s_{i}^{2} & \alpha_{1} c_{i} s_{i} \\
\alpha_{1}\left(1-\alpha_{2}\right) c_{i} s_{i} & \left(1-\alpha_{2}\right)\left(1-\alpha_{1} c_{i}^{2}\right)
\end{array}\right), \quad i \in 1, \ldots, p
$$


where $s_{i}:=\sin \theta_{i}, c_{i}:=\cos \theta_{i}$. The eigenvalues of $T$ are therefore $\lambda^{3}:=\left(1-\alpha_{2}\right)$, $\lambda^{4}:=\left(1-\alpha_{2}\right)\left(1-\alpha_{1}\right)$, and for every $T_{1}^{1}$

$$
\begin{aligned}
\lambda_{i}^{1,2}= & \frac{1}{2}\left(2-\alpha_{1}-\alpha_{2}+\alpha_{1} \alpha_{2} c_{i}^{2}\right) \\
& \pm \sqrt{\frac{1}{4}\left(2-\alpha_{1}-\alpha_{2}+\alpha_{1} \alpha_{2} c_{i}^{2}\right)^{2}-\left(1-\alpha_{1}\right)\left(1-\alpha_{2}\right)} .
\end{aligned}
$$

Remark 1 The property $p \leq q$ was used to arrive at these results. If instead $p>q$, we reverse the definitions of $P_{\mathcal{U}}$ and $P_{\mathcal{V}}$ in Proposition 1 . Noting that $\sigma(T)=\sigma\left(T^{T}\right)$, we get a new block-diagonal matrix $\bar{T}$ with blocks $\bar{T}_{1}=T_{1}^{T}, \bar{T}_{3}=T_{3}^{T}$ and $\bar{T}_{2}=\left(1-\alpha_{1}\right) I_{p-q}$. Therefore, the matrix will have eigenvalues in either $1-\alpha_{1}$ or $1-\alpha_{2}$ depending on the dimensions of $\mathcal{U}$ and $\mathcal{V}$.

Motivated by Fact 3 , we are looking for parameters that minimize the magnitude of the subdominant eigenvalues. We will do this for both cases in Remark 1 . In the following sequence of theorems, we will show that the optimal parameters are

$$
\alpha=1, \quad \alpha_{1}=\alpha_{2}=\alpha^{*}:=\frac{2}{1+\sin \theta_{F}},
$$

and that the subdominant eigenvalues have magnitude $\gamma(S)=\gamma^{*}$, where

$$
\gamma^{*}:=\frac{1-\sin \theta_{F}}{1+\sin \theta_{F}} .
$$

Theorem 1 The GAP operator $S$ in (2) with $\alpha, \alpha_{1}, \alpha_{2}$ as defined in (9) satisfies $\gamma(S)=\gamma^{*}$ and is linearly convergent with any rate $\mu \in\left(\gamma^{*}, 1\right)$.

A proof is located in Appendix A.1.

We now show that no other choices of $\alpha, \alpha_{1}, \alpha_{2}$ can achieve a lower linear convergence rate under the assumption that the relative dimension of $\mathcal{U}$ and $\mathcal{V}$ is unknown. Motivated by this, we formulate the following assumption.

Assumption 2 Suppose that $\mathcal{U}$ and $\mathcal{V}$ are linear subspaces and that the dimensions $p:=\operatorname{dim}(\mathcal{U}), q:=\operatorname{dim}(\mathcal{V})$ satisfy $p, q \in\{1, \ldots, n-1\}$ and consider the cases:

$$
\text { B1: } p<q, \quad \text { B2: } p=q \text {, and B3: } p>q \text {. }
$$

Proposition 2 To optimize the convergence rate of $S$, for all cases in Assumption 2 , it is necessary to minimize the largest modulus of the eigenvalues in the set

$$
\left(\left\{\lambda_{i}^{1,2}\right\}_{i \in 1, \ldots, p} \cap\left\{1-\alpha_{2}, 1-\alpha_{1},\left(1-\alpha_{2}\right)\left(1-\alpha_{1}\right)\right\}\right) \backslash\{1\} .
$$

Proof. These are the eigenvalues from the matrices in (6) together with $1-\alpha_{1}$, as motivated in Remark 1. If we let $\gamma_{1}=\gamma(S)$ under assumption B1, $\gamma_{2}=\gamma(S)$ under B2, and $\gamma_{3}=\gamma(S)$ under B3, it follows, from Remark 1, that the largest modulus of the eigenvalues in (11) is equal to $\max \left(\gamma_{1}, \gamma_{2}, \gamma_{3}\right)$.

Next, we show that the rate obtained in Theorem 1 is indeed optimal. 
Theorem 2 The GAP operator $S$ in (2) with $\theta_{F}<\pi / 2$ and $\alpha_{1}, \alpha_{2}, \alpha>0$ is linearly convergent with any rate $\mu \in\left(\gamma^{*}, 1\right)$, for all cases in Assumption 2 , if and only if $\alpha, \alpha_{1}, \alpha_{2}$ are chosen as in $(9)$.

A proof is located in Appendix A.3

Remark 2 The case with $\theta_{F}=\pi / 2$ is trivial and results in convergence in one iteration with the optimal parameters. This case is excluded from the theorem since there are also other methods that achieve the same rate. We also exclude the cases when either of $\alpha_{1}, \alpha_{2}, \alpha$ are non-positive, since such choices typically result in a nonconvergent algorithm. The assumption on the parameters is, however, less restrictive than Assumption 1.

Remark 3 The result is derived under the assumption that both $1-\alpha_{2}$ and $1-\alpha_{1}$ are considered, i.e. $q<p$ and $q>p$ respectively (see Remark 1). The same result follows in either of these cases if we instead assume that $\theta_{p}=\pi / 2$, which is a safe assumption if we do not know the largest principal angle.

We now state the convergence rate of the sequence $x^{k}$.

Theorem 3 The sequence $x^{k+1}:=S x^{k}$ with optimal parameters $\alpha=1, \alpha_{1}=\alpha_{2}=$ $\frac{2}{1+\sin \theta_{F}}$ converges linearly to $x^{*}:=P_{\text {fix } S} x^{0}$ according to

$$
\left\|x^{k}-x^{*}\right\| \leq \mu^{k}\left\|x^{0}\right\| \quad \forall k \geq N
$$

with any rate $\mu \in\left(\gamma^{*}, 1\right)$, for $\gamma^{*}$ in 10$)$, i.e., $x^{k}$ is $R$-linearly convergent to $x^{*}$.

A proof is located in Appendix A.4.

Remark 4 For linear subspaces $\mathcal{U}, \mathcal{V}$, under the Assumption 1 case A1 or A2, we have fix $S=\mathcal{U} \cap \mathcal{V}$, see e.g. [25]. For case A3 we have fix $S=\mathcal{V} \cap \mathcal{U}+\left(\mathcal{V}^{\perp} \cap \mathcal{U}^{\perp}\right)$, see 17 .

\section{Comparison with other choices of parameters}

In Section 3, we derive, for two linear subspaces, the optimal parameters for the generalized alternating projections method. These parameters are optimal under the assumption that the relative dimensions of the two subspaces are unknown, or that the largest principal angle $\theta_{p}=\pi / 2$. There are other methods that can perform better if these assumptions are not true. For example, if $\operatorname{dim} \mathcal{U} \leq \operatorname{dim} \mathcal{V}$, the parameters

$$
\alpha=1, \quad \alpha_{1}=2, \quad \alpha_{2}=\frac{2}{1+\sin \left(2 \theta_{F}\right)},
$$

(referred to as GAP2 $\alpha$ in Section 6) result in that most eigenvalues have modulus

$$
\frac{\cos \theta_{F}-\sin \theta_{F}}{\cos \theta_{F}+\sin \theta_{F}}
$$

This rate is better than $\gamma^{*}$, although marginally for small $\theta_{F}$. However, if the largest principal angle, $\theta_{p}$, is large enough, the corresponding eigenvalues will approach -1 . 


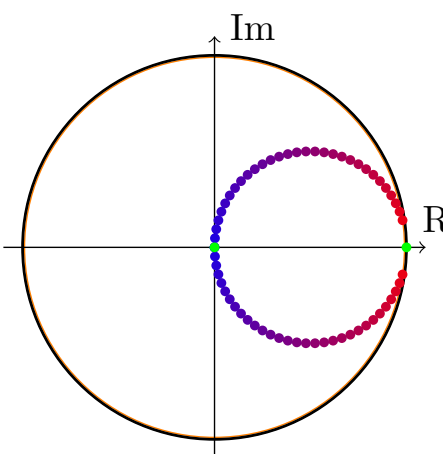

(a) Douglas-Rachford $\gamma(S) \approx 0.99$

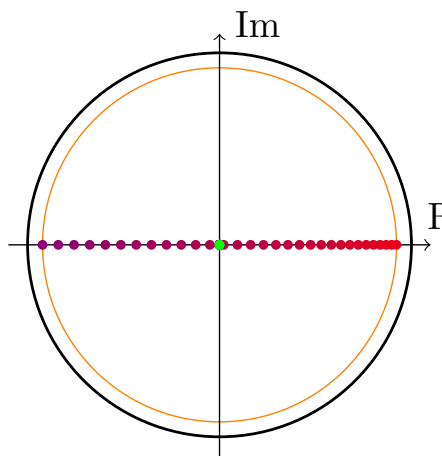

(d) PRAP 1.65

$\gamma(S) \approx 0.92$

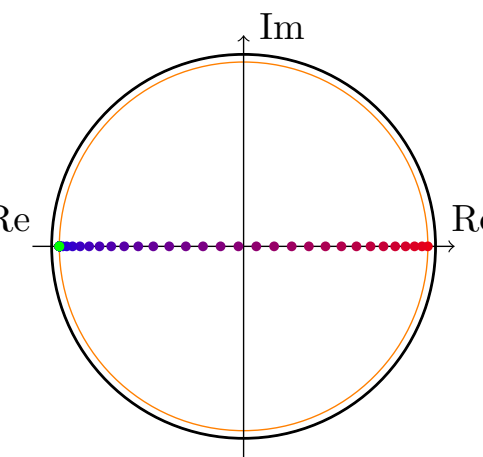

(b) MAP

$\gamma(S) \approx 0.96$

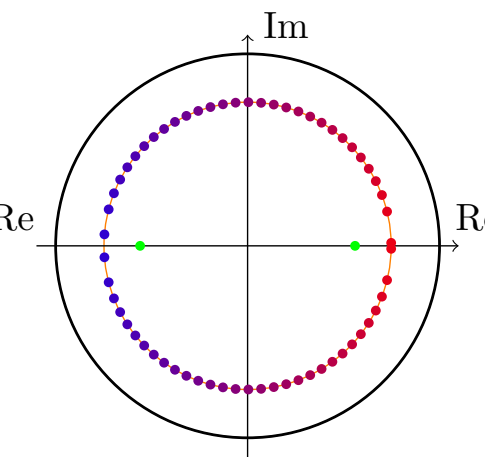

(e) GAP2 $\alpha$

$\gamma(S) \approx 0.748$

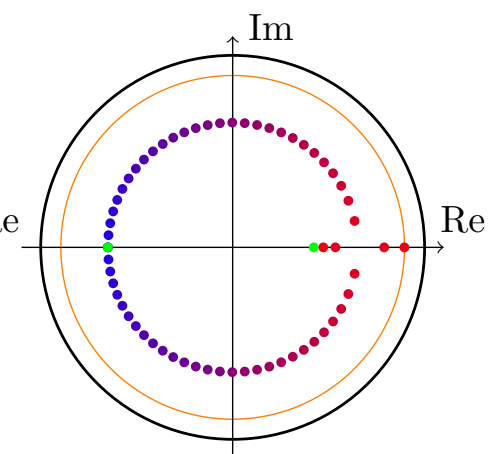

(c) GAP 1.65

$\gamma(S) \approx 0.90$

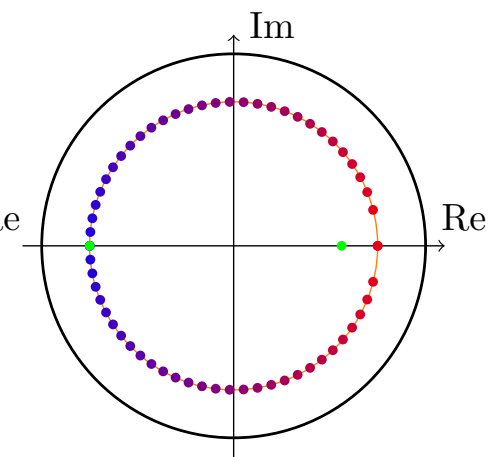

(f) $\mathrm{GAP}^{*}$

$\gamma(S) \approx 0.75$

Figure 1: Convergence rates for different methods, as described in Section 4, for $\theta_{F} \approx 0.14\left(8.8^{\circ}\right)$. The eigenvalues corresponding to the principal angels are shown for 30 angles, evenly spaced from $\theta_{F}$ to $\pi / 2$, as dots from red to blue. The eigenvalues corresponding to $\left(1-\alpha_{2}\right)$ and $\left(1-\alpha_{2}\right)\left(1-\alpha_{1}\right)$ are shown as green dots. The radius $\gamma(S)$ is shown in orange. GAP1.65 represents GAP with $\alpha=1$ and $\alpha_{1}=\alpha_{2}=1.65<$ $\alpha^{*}=1.75$. The partial relaxed alternating projections (PRAP) from Equation (15), the best algorithm in the previous work [18], is shown under the assumption $\theta_{p}=\pi / 4$. We see that the optimal parameters gives a much better result than the previously suggested methods. This is achieved by placing the eigenvalues at the same radius. Increasing the parameters from the optimal $\left(\alpha_{1}=\alpha_{2}>\alpha^{*}=1.75\right)$, increases the radius of the eigenvalues corresponding to the principal angles. If decreased, the result looks like GAP 1.65, where one of the eigenvalues corresponding to $\theta_{F}$ is subdominant. GAP2 $\alpha$ (Equation (13)) is shown under the assumption $\theta_{p} \approx 0.91 \pi / 2$. Although it performs slightly better than $\mathrm{GAP}^{*}$ under this assumtion, it gets considerably worse if $\theta_{p}$ increases. 
This choice will then converge much slower than the optimal method in Section 3 . This is observed in the numerical example in Section 6 .

When $\operatorname{dim} \mathcal{U} \leq \operatorname{dim} \mathcal{V}$, it is sometimes possible to get even better performance by selecting $\alpha_{2}>2$. However, this method is not convergent if $\operatorname{dim} \mathcal{U}>\operatorname{dim} \mathcal{V}$, and it would generally not be convergent for general convex sets.

In [18, optimal parameters are found by keeping two of the parameters fixed and optimizing over the third.

The first method is the relaxed alternating projections $\left(\alpha_{1}=\alpha_{2}=1\right)$, which is shown to be optimal for $\alpha=\frac{2}{1+\sin ^{2} \theta_{F}}$ with rate $\gamma=\left(1-\sin ^{2} \theta_{F}\right) /\left(1+\sin ^{2} \theta_{F}\right)$. This is better than the alternating projections with $\alpha=1$ which is convergent with rate $\gamma=\cos ^{2} \theta_{F}[16$.

The generalized Douglas-Rachford $\left(\alpha_{1}=\alpha_{2}=2\right)$, is shown to be optimal for $\alpha=0.5$ with rate $\gamma=\cos \theta_{F}$.

These rates are considerably worse than the optimal rates, as seen in Figure 1 . especially for small $\theta_{F}$. The methods are referred to as MAP and DR in the numerical example in Section 6 .

The partial relaxed alternating projections $\left(\alpha=\alpha_{2}=1\right)$ was was shown to be optimal for

$$
\alpha_{2}=\frac{2}{\sin ^{2} \theta_{p}+\sin ^{2} \theta_{F}}, \quad \text { with rate } \quad \gamma=\frac{\sin ^{2} \theta_{p}-\sin ^{2} \theta_{F}}{\sin ^{2} \theta_{p}+\sin ^{2} \theta_{F}} .
$$

This rate is sometimes better than $\gamma^{*}$ if $\theta_{p}<\pi / 2$, but not for small enough $\theta_{F}$. In fact, it is only better if $\sin ^{2} \theta_{p}<\sin \theta_{F}$. It also requires knowledge of $\theta_{p}$, and is not generally convergent if $\operatorname{dim} \mathcal{U}>\operatorname{dim} \mathcal{V}$.

An illustration of where the eigenvalues are located for these methods is shown in Figure 1 .

\section{$5 \quad$ Adaptive generalized alternating projections}

The generalized alternating projections algorithm with $\alpha_{1}=1, \alpha_{1}=\alpha_{2}=\frac{2}{1+\sin \theta_{F}}$ is optimal under the assumption that the relative dimensions between $\mathcal{U}$ and $\mathcal{V}$ is unknown. However, this parameter choice requires that the Friedrichs angle is known. This is typically not the case. In this section, we present an adaptive method that continuously tries to estimate the Friedrichs angle $\theta_{F}$ and updates $\alpha_{1}$ and $\alpha_{2}$, based on this estimate.

Consider the following estimate of the Friedrichs angle at iteration $k$

$$
\cos \hat{\theta}^{k}:=\frac{\left|\left\langle x^{k}-y^{k}, z^{k}-y^{k}\right\rangle\right|}{\left\|x^{k}-y^{k}\right\|\left\|z^{k}-y^{k}\right\|},
$$

where $y^{k}=P_{\mathcal{V}}^{\alpha_{1}} x^{k}$ and $z^{k}=P_{\mathcal{U}} P_{\mathcal{V}}^{\alpha_{1}} x^{k}$. If $x^{k}=y^{k}$ or $z^{k}=y^{k}$ we define the estimate as $\cos \theta_{k}:=0$. The estimate is illustrated in Figure 2

Next, we show that this value is always an overestimation of the Friedrichs angle, provided that the first iterate is in $\mathcal{U}+\mathcal{V}$.

Theorem 4 The estimate $\hat{\theta}^{k}$ in Equation (16) always satisfies $\hat{\theta}^{k} \geq \theta_{F}$ if the starting point $x^{0} \in \mathcal{U}+\mathcal{V}$. 


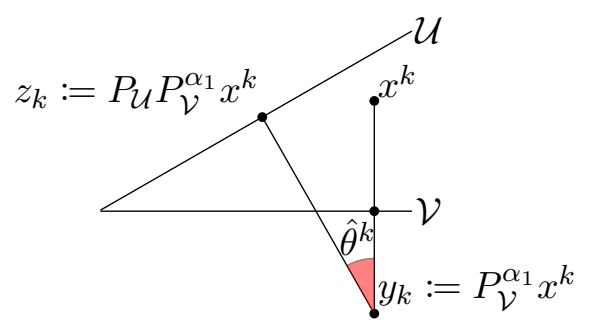

Figure 2: Illustration of the estimate $\hat{\theta}^{k}$.

Proof. Assume that $x^{k} \in \mathcal{U}+\mathcal{V}$. Since for a projection it holds that $P_{\mathcal{V}} x^{k} \in \mathcal{V}$, it follows that $y^{k}=P_{\mathcal{V}}^{\alpha_{1}} x^{k}$, a linear combination of $x^{k}$ and $P_{\mathcal{V}} x^{k}$, satisfies $y^{k} \in \mathcal{U}+\mathcal{V}$. In the same way it follows that $z^{k} \in \mathcal{U}+\mathcal{V}$ and $x^{k+1} \in \mathcal{U}+\mathcal{V}$. By induction, this must hold for all iterations since $x^{0} \in \mathcal{U}+\mathcal{V}$.

Let $v_{1}:=x^{k}-y^{k}$ and $v_{2}:=z^{k}-y^{k}$. We have $v_{1}=x^{k}-P_{\mathcal{V}}^{\alpha_{1}} x^{k}=\alpha_{1}\left(I-P_{\mathcal{V}}\right) x^{k}=$ $\alpha_{1} P_{\mathcal{V} \perp} x^{k} \in \mathcal{V}^{\perp}$ and in the same way $v_{2} \in \mathcal{U}^{\perp}$. We also see that $v_{1}, v_{2} \in \mathcal{U}+\mathcal{V}$, since they are linear combinations of elements in $\mathcal{U}+\mathcal{V}$. Noting that $\mathcal{U}+\mathcal{V}=\left(\mathcal{U}^{\perp} \cap \mathcal{V}^{\perp}\right)^{\perp}[16$, Lem. 2.11] we get,

$$
v_{1} \in \mathcal{U}^{\perp} \cap\left(\mathcal{U}^{\perp} \cap \mathcal{V}^{\perp}\right)^{\perp}, v_{2} \in \mathcal{V}^{\perp} \cap\left(\mathcal{U}^{\perp} \cap \mathcal{V}^{\perp}\right)^{\perp}
$$

Using the definition of the cosine of the Friedrichs angle between two sets $\mathcal{U}, \mathcal{V}[16$, Def. 2.1]:

$$
c_{F}(\mathcal{U}, \mathcal{V}):=\max \left\{\frac{|\langle v, u\rangle|}{\|v\|\|u\|}: \quad \begin{array}{ll} 
& v \in \mathcal{U} \cap(\mathcal{U} \cap \mathcal{V})^{\perp} \\
& u \in \mathcal{V} \cap(\mathcal{U} \cap \mathcal{V})^{\perp}
\end{array}\right\}
$$

and the property $c_{F}(\mathcal{U}, \mathcal{V})=c_{F}\left(\mathcal{U}^{\perp}, \mathcal{V}^{\perp}\right)[16$, Thm. 2.16] we immediately get

$$
\cos \hat{\theta}^{k}=\frac{\left|\left\langle v_{1}, v_{2}\right\rangle\right|}{\left\|v_{1}\right\|\left\|v_{2}\right\|} \leq c_{F}\left(\mathcal{U}^{\perp}, \mathcal{V}^{\perp}\right)=c_{F}(\mathcal{U}, \mathcal{V})=\cos \theta_{F}
$$

where we let $\frac{\left|\left\langle v_{1}, v_{2}\right\rangle\right|}{\left\|v_{1} \mid\right\| v_{2} \|}:=0$ if $\left\|v_{1}\right\|=0$ or $\left\|v_{2}\right\|=0$.

We therefore conclude that $\hat{\theta}^{k} \geq \theta_{F}$.

Next, we propose an adaptive version of the generalized alternating projections method:

Algorithm 1 Let $k=0, x^{0} \in \mathbb{R}^{n}$ and $\alpha^{0} \in(0,2)$.

$$
\begin{aligned}
y^{k} & :=P_{\mathcal{V}}^{\alpha^{k}} x^{k} \\
x^{k+1} & :=P_{\mathcal{U}}^{\alpha^{k}} y^{k} \\
\hat{\theta}^{k} & :=\operatorname{acos} \frac{\left|\left\langle x^{k}-y^{k}, x^{k+1}-y^{k}\right\rangle\right|}{\left\|x^{k}-y^{k}\right\|\left\|x^{k+1}-y^{k}\right\|} \\
\alpha^{k+1} & :=\frac{2}{1+\sin \hat{\theta}^{k}}
\end{aligned}
$$
$\mathcal{U}+\mathcal{V}$

We now motivate, without proof, that the estimate will tend toward $\theta_{F}$ if $x^{0} \in$

Let $\hat{\theta}^{k}$ be the current estimate of $\theta_{F}$ and $\alpha_{1}=\alpha_{2}=\frac{2}{1+\sin \hat{\theta}^{k}}$. Since $\hat{\theta}^{k} \geq \theta_{F}$, we get $\alpha_{1}=\alpha_{2} \leq \alpha^{*}$. As seen in Figure 1(c), eigenvalues corresponding to large principal 
angles have radius smaller than $\alpha^{*}-1$. However smaller principal angles will have one positive real eigenvalue, and the largest eigenvalue corresponds to $\theta_{F}$ with real part greater than $\alpha^{*}-1$. Iterating the operator should therefore result in convergence to the subspace spanned by the eigenvectors corresponding to $\theta_{F}$, and the estimated angle will decrease towards $\theta_{F}$. This behavior was observed in the numerical example in Section 6,

We now show that Algorithm 1 is always convergent, for general convex sets, if it is modified so that $\alpha^{k} \neq 2$. This is true if $\hat{\theta}_{F}>0$ or if the algorithm is modified, for example as

$$
\alpha^{k} \leftarrow \min \left\{\frac{2}{1+\sin \hat{\theta}^{k}}, 2-\epsilon\right\}
$$

for some $\epsilon>0$.

Theorem 5 Consider Algorithm 1 for two non-empty, closed, convex sets $\mathcal{U}, \mathcal{V}$ with $\mathcal{U} \cap \mathcal{V} \neq \emptyset$. If $\hat{\theta}^{k}$ satisfies $\hat{\theta}^{k}>0$ for all $k \geq 0$ then $x^{k} \rightarrow x^{*}$ for some $x^{*} \in \mathcal{U} \cap \mathcal{V}$.

Proof. If $\hat{\theta}^{k}>0$, then $\alpha^{k+1} \neq 2$. Thus $\alpha^{k+1} \in(0,2)$ and each iteration is the result of an averaged mapping $S^{k}$ with fixed points $\mathcal{U} \cap \mathcal{V}$. It follows that the iterates converge to the fixed point set $\mathcal{U} \cap \mathcal{V}$, see e.g. [25].

\section{$6 \quad$ Numerical Example}

In this section, we compare the theoretical results to numerical experiments. We have generated a set of problems of the form

$$
\mathcal{V}=\{x \mid A x=0\}, \mathcal{U}=\{x \mid B x=0\}
$$

with $A \in \mathbb{R}^{n \times 200}, B \in \mathbb{R}^{100 \times 200}$. The matrices are generated with independent normal distributed elements, with zero mean and unit variance. The initial point $x^{0}$ is randomly chosen in the same way. The dimension of $A$ is selected from 13 different categories with $n \in\{1, \ldots, 99\}$, and at least 500 problems are generated for each category, resulting in over 8000 different problems. The problems have Friedrichs angles in the range $\theta_{F} \in\left(5 \cdot 10^{-4}, 1\right)$.

We solve the problem of finding $x \in \mathcal{U} \cap \mathcal{V}$ using the following algorithms:

- Method of alternating projections (MAP):

$$
S_{\mathrm{MAP}}:=(1-\alpha) I+\alpha P_{\mathcal{V}} P_{\mathcal{U}}
$$

with optimal $\alpha=\frac{2}{1+\sin \left(\theta_{F}\right)^{2}}$, according to [18].

- Douglas-Rachford method (DR):

$$
S_{\mathrm{DR}}:=\frac{1}{2}\left(I+R_{\mathcal{V}} R_{\mathcal{U}}\right)
$$

where $R_{\mathcal{C}}:=P_{C}^{2}=2 P_{\mathcal{C}}-I$.

- The optimal generalized alternating projections $\left(\mathrm{GAP}^{*}\right)$ :

$$
S_{\mathrm{GAP}^{*}}:=P_{\mathcal{V}}^{\alpha^{*}} P_{\mathcal{U}}^{\alpha^{*}}
$$

with $\alpha^{*}=\frac{2}{1+\sin \theta_{F}}$. 


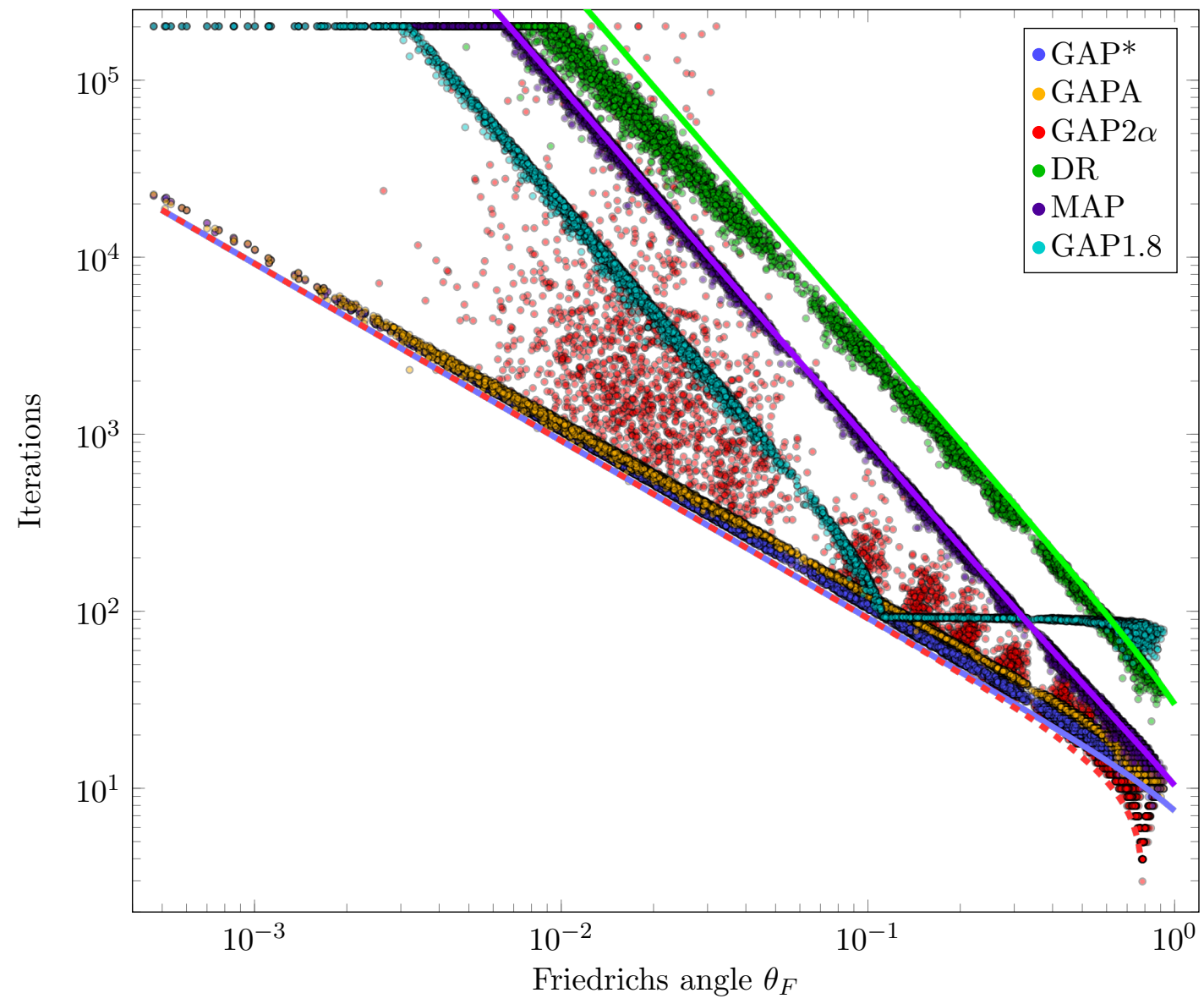

Figure 3: Number of iterations for different methods, as described in Section 6, plotted against the Friedrichs angle $\theta_{F}$. The theoretical rates are plotted in lines as the solution to $\gamma(S)^{n}=10^{-8}$ for GAP*, DR, and MAP. For GAP2 $\alpha$ we show the rate (in dashed red line) assuming that $\theta_{p}$ is sufficiently small, according to the discussion in Section 4. We see that this method can perform better than GAP*, particularly for large $\theta_{F}$. However, since $\theta_{p}$ is unknown, convergence is sometimes extremely slow. The convergence for GAP1.8 is constant for small $\theta_{F}$, but the convergence rate slows down considerably when $\theta_{F}$ decreases to the point where $1.8<\alpha^{*}$. We see that GAP* performs in line with the theoretical result, and considerably better than both DR and MAP. The adaptive method (GAPA) performs marginally worse than GAP* for large $\theta_{F}$. No difference in the number of iterations can be seen between GAP* and GAPA when $\theta_{F}$ is small. 
- The adaptive generalized alternating projections (GAPA):

$$
S_{\mathrm{GAPA}}:=P_{\mathcal{V}}^{\alpha_{k}} P_{\mathcal{U}}^{\alpha_{k}},
$$

implemented as in Algorithm 1

- Generalized alternating projections with $a=1, \alpha_{1}=2, \alpha_{2}=\frac{2}{1+\sin \left(2 \theta_{F}\right)}(\operatorname{GAP} 2 \alpha)$ :

$$
S_{\mathrm{GAP} 2 \alpha}=P_{\mathcal{V}}^{\alpha_{2}} R_{\mathcal{U}}
$$

as described in Section 4 .

- Generalized alternating projections with $\alpha=1, \alpha_{1}=\alpha_{2}=1.8$ (GAP1.8):

$$
S_{\mathrm{GAP} 1.8}:=P_{\mathcal{V}}^{1.8} P_{\mathcal{U}}^{1.8}
$$

For each of the methods we monitor the shadow sequence

$$
z^{k}=P_{\mathcal{U}} S^{k} x_{0}
$$

and terminate when

$$
\left\|P_{\mathcal{V} \cap \mathcal{U}} z^{k}-z^{k}\right\|<10^{-8}
$$

or when the number of iterations reach 200,000 .

Remark 5 The analysis in this paper concerns the convergence of the sequence towards a fixed-point. We are actually more interested in the shadow sequence (that we monitor in the examples), since it can find a point in the intersection long before the sequence converges to the fixed-point set. This may be favorable for the DouglasRachford algorithm because of its dominating complex eigenvalues, compared to what its convergence rate suggests.

The problems were generated and solved with Julia [26, and the results are shown in Figure 3. We see that the methods perform in line with the theoretical rates. The method with optimal parameters performs considerably better and more reliably than for other choices. We see that the adaptive method performs almost identically to the optimal parameters, without prior knowledge of the Friedrichs angle.

We have verified numerically that the estimate in the adaptive method converges to the Friedrichs angle. For all problems that took more than 17 iterations to converge, the estimate in the last iteration, was indeed conservative $\left(\hat{\theta}^{k}>\theta_{F}\right)$. Furthermore, the relative error $\left|\hat{\theta}^{k}-\theta_{F}\right| /\left\|\theta_{F}\right\|$ was smaller than $5 \%(0.1 \%)$ at the last iteration, for all problems that ran more than 100 (400) iterations. These results were obtained, even though no measures were taken to ensure $x^{0} \in \mathcal{U}+\mathcal{V}$.

\section{Conclusions}

We derived the optimal parameters for the generalized alternating projections method for two linear subspaces. The optimal rate is considerably better than previously analyzed parameters, and we verify the results with an extensive set of numerical examples. We also presented an adaptive method, that in practice is able to perform in line with the optimal parameters, with no prior knowledge about the problem.

It remains as future work to study how the results apply to more general feasibility problems. 


\section{A Appendix}

\section{A.1 Proof of Theorem 1}

We divide the proof into two cases: $p+q<n$ and $p+q \geq n$.

Case $1(p+q<n)$ For this case we can use the results in Proposition 1. For convenience of notation we introduce

$$
\begin{aligned}
& f(\theta):=\frac{1}{2}\left(2-\alpha_{1}-\alpha_{2}+\alpha_{1} \alpha_{2} \cos ^{2} \theta\right) \\
& g(\theta):=\sqrt{f(\theta)^{2}-\left(1-\alpha_{1}\right)\left(1-\alpha_{2}\right)}
\end{aligned}
$$

so that 8 becomes $\lambda_{i}^{1,2}=f\left(\theta_{i}\right) \pm g\left(\theta_{i}\right)$. For $\alpha_{1}=\alpha_{2}=\alpha^{*}=\frac{2}{1+\sin \theta_{F}}$ we get $f\left(\theta_{F}\right)=$ $1-\alpha^{*}+\alpha^{* 2} c_{F}^{2}=\frac{1-\sin \theta_{F}}{1+\sin \theta_{F}}=\alpha^{*}-1$ and $g\left(\theta_{F}\right)=0$. Therefore, $\lambda_{F}^{1,2}=\alpha^{*}-1=\frac{1-\sin \theta_{F}}{1+\sin \theta_{F}}$. We also see that $f(\pi / 2)=1-\alpha^{*}, g(\pi / 2)=0$. Since $f(\theta)$ is linear in $\cos ^{2} \theta$ and $\left|f\left(\theta_{F}\right)\right|=|f(\pi / 2)|=\alpha^{*}-1$, it follows that $\left|f\left(\theta_{i}\right)\right| \leq \alpha^{*}-1$ for all $\theta_{i} \in\left[\theta_{F}, \pi / 2\right]$. This means that the corresponding $\lambda_{i}^{1,2}$ are complex with amplitudes

$$
\begin{aligned}
\left|\lambda_{i}^{1,2}\right| & =\sqrt{f\left(\theta_{i}\right)^{2}+\left|f\left(\theta_{i}\right)^{2}-\left(1-\alpha^{*}\right)^{2}\right|}=\sqrt{\left(1-\alpha^{*}\right)^{2}} \\
& =\alpha^{*}-1 \quad \forall i: \theta_{F} \leq \theta_{i} \leq \pi / 2 .
\end{aligned}
$$

Lastly, for $\theta_{i}<\theta_{F}$, we have $\theta_{i}=0$ and $\lambda_{i}^{1,2}=\left\{1,\left(1-\alpha^{*}\right)^{2}\right\}$ as seen in Equation (7). To conclude, we have

$$
\begin{aligned}
\left|\lambda_{F}^{1,2}\right| & =\left|\lambda^{3}\right|=\alpha^{*}-1 \\
\left|\lambda^{4}\right| & =\left(\alpha^{*}-1\right)^{2}<\alpha^{*}-1 \\
\left|\lambda_{i}^{1,2}\right| & =\alpha^{*}-1 \quad \forall i: \theta_{F} \leq \theta_{i} \leq \pi / 2 \\
\left|\lambda_{i}^{1,2}\right| & =\left\{1,\left(1-\alpha^{*}\right)^{2}\right\} \quad \forall i: \theta_{i}<\theta_{F},
\end{aligned}
$$

where $\lambda^{3}$ corresponds to eigenvalues in $1-\alpha_{2}$ and $1-\alpha_{1}$ in Proposition 2. The eigenvalues $\lambda=1$ are semisimple since the matrix in (7) is diagonal for $\theta_{i}=0$. We therefore conclude, from Fact 2 and 3 , that $\alpha_{1}=\alpha_{2}=\alpha^{*}$ results in that $T=S$ in Equation (2) is linearly convergent with any rate $\mu \in\left(\gamma^{*}, 1\right)$ where $\gamma^{*}=\alpha^{*}-1=$ $\frac{1-\sin \theta_{F}}{1+\sin \theta_{F}}$ is a subdominant eigenvalue.

Case $2(p+q \geq n)$ The following proof follows closely that in [17, p. 54]. We can extend the space $\mathbb{R}^{n}$ with $k$ extra dimensions so that $p+q<n+k=: \bar{n}$. Let $\overline{\mathcal{U}}:=\mathcal{U} \times\left\{0_{k}\right\}, \overline{\mathcal{V}}:=\mathcal{V} \times\left\{0_{k}\right\}$, and therefore

$$
P_{\overline{\mathcal{U}}}=\left(\begin{array}{cc}
P_{\mathcal{U}} & 0 \\
0 & 0_{k}
\end{array}\right), \quad P_{\overline{\mathcal{V}}}=\left(\begin{array}{cc}
P_{\mathcal{V}} & 0 \\
0 & 0_{k}
\end{array}\right) .
$$

It follows that

$$
\bar{S}=\bar{T}:=P_{\overline{\mathcal{U}}}^{\alpha^{*}} P_{\overline{\mathcal{V}}}^{\alpha^{*}}=\left(\begin{array}{cc}
T & 0 \\
0 & \left(1-\alpha^{*}\right)^{2} I_{k}
\end{array}\right),
$$


where $S=T=P_{\mathcal{U}}^{\alpha^{*}} P_{\mathcal{V}}^{\alpha^{*}}$. By using Case 1 on this matrix we conclude that $\gamma(\bar{S})=\gamma^{*}$. From their definition, we see that the principal angles between $\mathcal{U}$ and $\mathcal{V}$ are the same as the ones between $\overline{\mathcal{U}}$ and $\overline{\mathcal{V}}$. Since $\bar{T}$ is block diagonal we have $\sigma(\bar{T})=\sigma(T) \cup\left\{\left(1-\alpha^{*}\right)^{2}\right\}$. We therefore know that the eigenvalues $\lambda_{F}^{1,2}=\alpha^{*}-1$ in $\bar{T}$, corresponding to the Friedrichs angle, must also be in $T$. This means that $\gamma(S) \geq \alpha^{*}-1=\gamma^{*}$. But $\left\|S^{k}-S^{\infty}\right\| \leq\left\|\bar{S}^{k}-\bar{S}^{\infty}\right\|$ so from Fact 3 we know that $\gamma(S) \leq \gamma(\bar{S})=\gamma^{*}$. We have therefore shown that $\gamma(S)=\gamma^{*}$, and the proof is complete.

\section{A.2 Lemmas}

Lemma 1 The matrix $M:=\left(2-\alpha^{*}\right) I+\frac{\alpha^{*}}{\alpha_{1}}\left(T_{1}^{i}-I\right)$ where $T_{1}^{i}$ is the matrix (7) corresponding to the angle $\theta_{F}$ has trace and determinant:

$$
\begin{aligned}
\operatorname{tr} M & =\frac{2}{(1+s) \alpha_{1}}\left(-\alpha_{1}-\alpha_{2}+\alpha_{2} \alpha_{1} c^{2}+2 \alpha_{1} s\right) \\
\operatorname{det} M & =\frac{4 s(1-s)}{\alpha_{1}(1+s)^{2}}\left(-\alpha_{1}-\alpha_{2}+\alpha_{1} \alpha_{2}(1+s)\right),
\end{aligned}
$$

where $s:=\sin \theta_{F}, c:=\cos \theta_{F}$.

Proof. Let $s:=\sin \theta_{F}, c:=\cos \theta_{F}$. The matrix can be written

$$
\begin{aligned}
M & \left.=\left(2-\alpha^{*}\right) I+\frac{\alpha^{*}}{\alpha_{1}}\left(\begin{array}{cc}
1-\alpha_{1} s^{2} & \alpha_{1} c s \\
\alpha_{1}\left(1-\alpha_{2}\right) c s & \left(1-\alpha_{2}\right)\left(1-\alpha_{1} c^{2}\right)
\end{array}\right)-I\right) \\
& =\left(\begin{array}{cc}
2-\alpha^{*}-\alpha^{*} s^{2} & \alpha^{*} c s \\
\alpha^{*}\left(1-\alpha_{2}\right) c s & 2-\alpha^{*}+\frac{\alpha^{*}}{\alpha_{1}}\left(\left(1-\alpha_{2}\right)\left(1-\alpha_{1} c^{2}\right)-1\right)
\end{array}\right) \\
& =\left(\begin{array}{cc}
2-\alpha^{*}\left(1+s^{2}\right) & \alpha^{*} c s \\
\alpha^{*}\left(1-\alpha_{2}\right) c s & 2-\alpha^{*}+\frac{\alpha^{*}}{\alpha_{1}}\left(\alpha_{1} \alpha_{2} c^{2}-\alpha_{2}-\alpha_{1} c^{2}\right)
\end{array}\right) .
\end{aligned}
$$

Using that $\alpha^{*}=\frac{2}{1+s}$, we can rewrite the diagonal elements

$$
2-\alpha^{*}\left(1+s^{2}\right)=\alpha^{*}\left(1+s-\left(1+s^{2}\right)\right)=\alpha^{*} s(1-s)
$$

and

$$
\begin{aligned}
2-\alpha^{*} & +\frac{\alpha^{*}}{\alpha_{1}}\left(\alpha_{1} \alpha_{2} c^{2}-\alpha_{2}-\alpha_{1} c^{2}\right)= \\
& =\alpha^{*}(1+s)-\alpha^{*}+\alpha^{*}\left(c^{2}\left(\alpha_{2}-1\right)-\frac{\alpha_{2}}{\alpha_{1}}\right) \\
& =\alpha^{*}\left(s+c^{2}\left(\alpha_{2}-1\right)-\frac{\alpha_{2}}{\alpha_{1}}\right) .
\end{aligned}
$$

We can extract the factor $\alpha^{*} c s$ from the matrix and get

$$
M=\alpha^{*} c s\left(\begin{array}{cc}
\frac{1-s}{c} & 1 \\
1-\alpha_{2} & \frac{s+c^{2}\left(\alpha_{2}-1\right)-\frac{\alpha_{2}}{\alpha_{1}}}{c s}
\end{array}\right) .
$$


The trace is therefore given by

$$
\begin{aligned}
\operatorname{tr} M & =\alpha^{*} c s\left(\frac{1-s}{c}+\frac{s+c^{2}\left(\alpha_{2}-1\right)-\frac{\alpha_{2}}{\alpha_{1}}}{c s}\right) \\
& =\alpha^{*}\left(2 s-s^{2}+c^{2} \alpha_{2}-c^{2}-\frac{\alpha_{2}}{\alpha_{1}}\right) \\
& =\frac{\alpha^{*}}{\alpha_{1}}\left(-\alpha_{1}-\alpha_{2}+\alpha_{2} \alpha_{1} c^{2}+2 \alpha_{1} s\right) \\
& =\frac{2}{(1+s) \alpha_{1}}\left(-\alpha_{1}-\alpha_{2}+\alpha_{2} \alpha_{1} c^{2}+2 \alpha_{1} s\right)
\end{aligned}
$$

and the determinant

$$
\begin{aligned}
\operatorname{det} M & =\left(\alpha^{*} c s\right)^{2}\left(\frac{(1-s)\left(s+c^{2}\left(\alpha_{2}-1\right)-\frac{\alpha_{2}}{\alpha_{1}}\right)}{c^{2} s}-\frac{\left(1-\alpha_{2}\right) c^{2} s}{c^{2} s}\right) \\
& =\alpha^{* 2} s\left(\left(s+c^{2}\left(\alpha_{2}-1\right)-\frac{\alpha_{2}}{\alpha_{1}}-s^{2}-c^{2} s\left(\alpha_{2}-1\right)+s \frac{\alpha_{2}}{\alpha_{1}}\right)-\left(1-\alpha_{2}\right) c^{2} s\right) \\
& =\alpha^{* 2} s\left(s+c^{2}\left(\alpha_{2}-1\right)-\frac{\alpha_{2}}{\alpha_{1}}-s^{2}+s \frac{\alpha_{2}}{\alpha_{1}}\right) \\
& =\alpha^{* 2} s\left(s-1+\alpha_{2} c^{2}+\frac{\alpha_{2}}{\alpha_{1}}(s-1)\right) \\
& =\alpha^{* 2} s(1-s)\left(-1+\alpha_{2}(1+s)-\frac{\alpha_{2}}{\alpha_{1}}\right) \\
& =\frac{\alpha^{* 2} s(1-s)}{\alpha_{1}}\left(-\alpha_{1}-\alpha_{2}+\alpha_{1} \alpha_{2}(1+s)\right) \\
& =\frac{4 s(1-s)}{\alpha_{1}(1+s)^{2}}\left(-\alpha_{1}-\alpha_{2}+\alpha_{1} \alpha_{2}(1+s)\right)
\end{aligned}
$$

Lemma 2 Under the assumptions $\alpha=\frac{\alpha^{*}}{\alpha_{1}}, \alpha_{1} \geq \alpha_{2}>0$ and $\theta_{F}<\pi / 2$, then, the matrix $M$ in Lemma 1 satisfies

$$
\left(\alpha_{1} \neq \alpha^{*} \text { or } \alpha_{2} \neq \alpha^{*}\right) \Rightarrow \max \operatorname{Re} \sigma(M)>0 .
$$

Proof. We show the equivalent claim

$$
\max \operatorname{Re} \sigma(M) \leq 0 \Rightarrow \alpha_{1}=\alpha_{2}=\alpha^{*} .
$$

We have $\max \operatorname{Re} \sigma(M) \leq 0$ if and only if both eigenvalues of $M$ have negative or zero real part, which is equivalent to

$$
\lambda_{1}+\lambda_{2} \leq 0 \text { and } \lambda_{1} \lambda_{2} \geq 0
$$

which is equivalent to

$$
\operatorname{tr} M \leq 0 \text { and } \operatorname{det} M \geq 0
$$


Using Lemma 1, this can be written

$$
\left\{\begin{array}{ll}
\frac{2}{(1+s) \alpha_{1}}\left(-\alpha_{1}-\alpha_{2}+\alpha_{2} \alpha_{1} c^{2}+2 \alpha_{1} s\right) & \leq 0 \\
\frac{4 s(1-s)}{\alpha_{1}(1+s)^{2}}\left(-\alpha_{1}-\alpha_{2}+\alpha_{1} \alpha_{2}(1+s)\right) & \geq 0
\end{array},\right.
$$

where $s:=\sin \left(\theta_{F}\right)$ and $c:=\cos \left(\theta_{F}\right)$. Since $\alpha_{1}>0, s \in(0,1)$, this is equivalent to

$$
\begin{cases}\alpha_{1}+\alpha_{2}-\alpha_{2} \alpha_{1} c^{2}-2 \alpha_{1} s & \geq 0 \\ -\alpha_{1}-\alpha_{2}+\alpha_{1} \alpha_{2}(1+s) & \geq 0\end{cases}
$$

This implies that the sum is positive, i.e.

$$
\begin{gathered}
\left(\alpha_{1}+\alpha_{2}-\alpha_{2} \alpha_{1} c^{2}-2 \alpha_{1} s\right)+\left(-\alpha_{1}-\alpha_{2}+\alpha_{1} \alpha_{2}(1+s)\right) \\
=\left(\alpha_{2} \alpha_{1} s^{2}-2 \alpha_{1} s+\alpha_{1} \alpha_{2} s\right) \\
\quad=\alpha_{1} s\left(\alpha_{2} s-2+\alpha_{2}\right) \geq 0 \\
\Leftrightarrow \quad \alpha_{2}(1+s) \geq 2 \\
\Leftrightarrow \quad \alpha_{2} \geq \frac{2}{1+s}=\alpha^{*}
\end{gathered}
$$

But then, 19a implies

$$
\begin{gathered}
\alpha_{1}+\alpha_{2}-\alpha_{2} \alpha_{1} c^{2}-2 \alpha_{1} s \geq 0 \\
\Rightarrow\left(\text { since } \alpha_{2} \geq \alpha^{*}\right) \\
\alpha_{1}+\alpha_{2}-\alpha^{*} \alpha_{1} c^{2}-2 \alpha_{1} s \geq 0
\end{gathered}
$$

which is equivalent to

$$
\begin{aligned}
\alpha_{1}+\alpha_{2}-\alpha^{*} \alpha_{1} c^{2}-2 \alpha_{1} s & = \\
\alpha_{1}+\alpha_{2}-2 \alpha_{1}(1-s)-2 \alpha_{1} s & = \\
\alpha_{1}+\alpha_{2}-2 \alpha_{1} & = \\
\alpha_{2}-\alpha_{1} & \geq 0
\end{aligned}
$$

i.e.

$$
\alpha_{2} \geq \alpha_{1} .
$$

But by assumption $\alpha_{1} \geq \alpha_{2}$ so we know that (19) implies $\alpha_{1}=\alpha_{2} \geq \alpha^{*}$. Equation 19a yields

$$
\begin{aligned}
& \alpha_{1}+\alpha_{2}-\alpha_{2} \alpha_{1} c^{2}-2 \alpha_{1} s \geq 0 \\
& \Rightarrow \quad 2 \alpha_{1}-\alpha_{1}^{2} c^{2}-2 \alpha_{1} s \geq 0 \\
& \Leftrightarrow \quad 2-\alpha_{1} c^{2}-2 s \geq 0 \\
& \Leftrightarrow \quad 2 \frac{(1-s)}{c^{2}} \geq \alpha_{1} \\
& \Leftrightarrow \quad \alpha^{*}=\frac{2}{(1+s)} \geq \alpha_{1} .
\end{aligned}
$$

where the implication is from $\alpha_{1}=\alpha_{2}$. We have therefore shown that $\alpha^{*} \geq \alpha_{1}=\alpha_{2} \geq$ $\alpha^{*}$ i.e.

$$
\max \operatorname{Re} \sigma(M) \leq 0 \Rightarrow \alpha_{1}=\alpha_{2}=\alpha^{*}
$$




\section{A.3 Proof of Theorem 2}

The first direction is proven by Theorem 1. To prove the other direction, we consider two cases.

Case $1(p+q<n)$ Assume that $\alpha_{1} \geq \alpha_{2}$ and let $\alpha=\hat{\alpha}:=\frac{\alpha^{*}}{\alpha_{1}}$. The eigenvalues to the GAP operator $S$ in (1) are $1+\alpha(\lambda-1)$ where $\lambda$ are the eigenvalues to $T$. Motivated by Proposition 2, we need to consider the following eigenvalues

$$
\left\{\lambda_{i}^{1,2}\right\}_{i \in 1, \ldots, p} \cap\left\{1-\alpha_{2}, 1-\alpha_{1},\left(1-\alpha_{2}\right)\left(1-\alpha_{1}\right)\right\} .
$$

For the eigenvalue $\lambda=1-\alpha_{1}$, we get

$$
1+\hat{\alpha}(\lambda-1)=1+\frac{\alpha^{*}}{\alpha_{1}}\left(1-\alpha_{1}-1\right)=1-\alpha^{*} .
$$

We now show that all choices of $\alpha, \alpha_{1}, \alpha_{2}$ results in an eigenvalue with real part larger than $\alpha^{*}-1$ unless $\alpha=1, \alpha_{1}=\alpha_{2}=\alpha^{*}$.

Consider the eigenvalues to $I+\hat{\alpha}\left(T_{1}^{i}-I\right)$ where $T_{1}^{i}$ is the matrix (7) corresponding to the angle $\theta_{F}$. We have

$$
\max \operatorname{Re} \sigma\left(I+\hat{\alpha}\left(T_{1}^{i}-I\right)\right)>\alpha^{*}-1
$$

if and only if

$$
\max \operatorname{Re} \sigma\left(\left(2-\alpha^{*}\right) I+\hat{\alpha}\left(T_{1}^{i}-I\right)\right)>0 .
$$

By Lemma 2 we know that (22) is true when $\alpha=\hat{\alpha}$, unless $\alpha_{1}=\alpha_{2}=\alpha^{*}$. We therefore know that for $\alpha=\hat{\alpha}$, unless the optimal parameters are selected, there will always be one eigenvalue of $S$ in $1-\alpha^{*}$ and one, corresponding to $\theta_{F}$, with real part greater than $\alpha^{*}-1$. We now consider the two cases $\alpha>\hat{\alpha}$ and $\alpha<\hat{\alpha}$. First note that $\alpha$ acts as a scaling of the eigenvalues relative to the point 1, i.e. $(1-\alpha)+\alpha \lambda=1+\alpha(\lambda-1)$. It is therefore clear that $\alpha>\hat{\alpha}$ will result in one eigenvalue with real part less than $1-\alpha^{*}=-\gamma^{*}$, and thus $\gamma(S)>\gamma^{*}$.

Similarly, any $\alpha<\hat{\alpha}$ will result in one eigenvalue $\left(\lambda_{F}^{1}\right)$ with real part greater than $\alpha^{*}-1=\gamma^{*}$. If this eigenvalue is not in 1, i.e. unless $1+\alpha\left(\lambda_{F}^{1}-1\right)=1$, we know that $\gamma(S)>\gamma^{*}$ also in this case. Since $\alpha \neq 0$ we have $1+\alpha\left(\lambda_{F}^{1}-1\right)=1$ if and only if $\lambda_{F}^{1}=1$. But $\lambda_{F}^{1}=1$ only if $\operatorname{det}\left(T_{F}-I\right)=0$, where $T_{F}$ is the block corresponding to $\theta_{F}$ in Equation (7). Since $\alpha_{1}, \alpha_{2} \neq 0$ and $\theta_{F}>0$ we get

$$
\operatorname{det}\left(T_{F}-I\right)=-\alpha_{1} s_{F}^{2}\left(\alpha_{1} c_{F}^{2}-\alpha_{2}+\alpha_{1} \alpha_{2} c_{F}^{2}\right)-\alpha_{1}^{2}\left(1-\alpha_{2}\right) c_{F}^{2} s_{F}^{2}=\alpha_{1} \alpha_{2} s_{F}^{2} \neq 0
$$

and thus $\lambda_{F}^{1} \neq 1$.

We conclude that when $\alpha_{1}>\alpha_{2}$, then $\gamma(S)>\alpha^{*}-1$ for all parameters that are not $\alpha=1, \alpha_{1}=\alpha_{2}=\alpha^{*}$.

The proof is only dependent on the location of the eigenvalue corresponding to $\theta_{F}$ and the one in $1-\alpha_{1}$ from Proposition 2. From symmetry of $\alpha_{1}, \alpha_{2}$ in (8) we see that the same argument holds if we instead assume $\alpha_{2} \geq \alpha_{1}$, let $\hat{\alpha}=\alpha^{*} / \alpha_{2}$, and pick the eigenvalue $1-\alpha_{2}$, from the set in Proposition 2, in (20) instead. We have therefore shown that $\gamma(S)>\alpha^{*}-1$ unless $\alpha=1, \alpha_{1}=\alpha_{2}=\alpha^{*}$ so the result follows from Fact 3 .

Case $2(p+q \geq n)$ As in the proof of Theorem 1 we can extend the space $\mathbb{R}^{n}$ with $k$ extra dimensions so that $p+q<n+k=: \bar{n}$. With $\overline{\mathcal{U}}:=\mathcal{U} \times\left\{0_{k}\right\}, \overline{\mathcal{V}}:=\mathcal{V} \times\left\{0_{k}\right\}$ 
we get, as in the proof for Theorem 1, that $\sigma(\bar{T})=\sigma(T) \cup\left\{\left(1-\alpha_{1}\right)\left(1-\alpha_{2}\right)\right\}$ and that the principal angles between $\mathcal{U}$ and $\mathcal{V}$ are the same as between $\overline{\mathcal{U}}$ and $\overline{\mathcal{V}}$. Using Proposition 1 on $\bar{T}$ we therefore see that the eigenvalues corresponding to the Friedrichs angle will exist in both $\bar{T}$ and $T$. The same is true for the eigenvalues in $1-\alpha_{2}$ and $1-\alpha_{1}$ for the respective cases $B 1$ and $B 3$ in Assumption 2. The proof in Case 1 can therefore be used to show that $\gamma(S)>\gamma^{*}$ unless $\alpha=1, \alpha_{1}=\alpha_{2}=\alpha^{*}$.

\section{A.4 Proof of Theorem 3}

Using [18, Thm. 2.12] we get for convergent $A$ :

$$
\begin{aligned}
\left\|x^{k}-x^{*}\right\| & =\left\|A^{k} x^{0}-A^{\infty} x^{0}\right\|=\left\|\left(A^{k}-A^{\infty}\right) x^{0}\right\| \\
& =\left\|\left(A-A^{\infty}\right)^{k} x_{0}\right\| \leq\left\|\left(A-A^{\infty}\right)^{k}\right\|\left\|x_{0}\right\| .
\end{aligned}
$$

Using the spectral radius formula and $\rho\left(A-A^{\infty}\right)=\gamma(A)$ [18, Thm. 2.12] we have, for any $\mu \in(\gamma(A), 1)$

$$
\lim _{k \rightarrow \infty}\left\|\left(A-A^{\infty}\right)^{k}\right\|^{\frac{1}{k}}=\rho\left(A-A^{\infty}\right)=\gamma(A)<\mu,
$$

so there exists $N \in \mathbb{N}$ such that $\left\|\left(A-A^{\infty}\right)^{k}\right\| \leq \mu^{k}, \forall k \geq N$ and thus

$$
\left\|x^{k}-x^{*}\right\| \leq \mu^{k}\left\|x^{0}\right\| \quad \forall k \geq N .
$$

From [18, Corollary 2.7] we know that $S^{\infty}=P_{\text {fix } S}$ since $S$ is nonexpansive, we therefore get $x^{*}=P_{\mathrm{fix} S} x^{0}$.

From Theorem 1 we know that $\gamma(S)=\frac{1-\sin \theta_{F}}{1+\sin \theta_{F}}$, and the proof is complete.

\section{References}

[1] J. von Neumann, Functional Operators. Volume II. The Geometry of Orthogonal Spaces. Princeton University Press: Annals of Mathematics Studies, 1950. Reprint of 1933 lecture notes.

[2] F. Deutsch, The Method of Alternating Orthogonal Projections, pp. 105-121. Dordrecht: Springer Netherlands, 1992.

[3] S. Agmon, "The relaxation method for linear inequalities," Canadian Journal of Mathematics, vol. 6, no. 3, pp. 382-392, 1954.

[4] T. S. Motzkin and I. Shoenberg, "The relaxation method for linear inequalities," Canadian Journal of Mathematics, vol. 6, no. 3, pp. 383-404, 1954.

[5] L. M. Bregman, "Finding the common point of convex sets by the method of successive projection," Dokl Akad. Nauk SSSR, vol. 162, no. 3, pp. 487-490, 1965.

[6] J. P. Boyle and R. L. Dykstra, A Method for Finding Projections onto the Intersection of Convex Sets in Hilbert Spaces, pp. 28-47. New York, NY: Springer New York, 1986. 
[7] J. Douglas and H. H. Rachford, "On the numerical solution of heat conduction problems in two and three space variables," Trans. Amer. Math. Soc., vol. 82, pp. 421-439, 1956.

[8] P. L. Lions and B. Mercier, "Splitting algorithms for the sum of two nonlinear operators," SIAM Journal on Numerical Analysis, vol. 16, no. 6, pp. 964-979, 1979.

[9] R. Glowinski and A. Marroco, "Sur l'approximation, par éléments finis d'ordre un, et la résolution, par pénalisation-dualité d'une classe de problémes de dirichlet non linéaires," ESAIM: Mathematical Modelling and Numerical Analysis Modélisation Mathématique et Analyse Numérique, vol. 9, pp. 41-76, 1975.

[10] S. Boyd, N. Parikh, E. Chu, B. Peleato, and J. Eckstein, "Distributed optimization and statistical learning via the alternating direction method of multipliers," Foundations and Trends in Machine Learning, vol. 3, no. 1, pp. 1-122, 2011.

[11] H. M. Phan, "Linear convergence of the douglas-rachford method for two closed sets," Optimization, vol. 65, no. 2, pp. 369-385, 2016.

[12] R. Hesse and D. R. Luke, "Nonconvex notions of regularity and convergence of fundamental algorithms for feasibility problems," SIAM Journal on Optimization, vol. 23, no. 4, pp. 2397-2419, 2013.

[13] D. Davis and W. Yin, "Faster convergence rates of relaxed Peaceman-Rachford and ADMM under regularity assumptions." Available: http://arxiv.org/abs/ 1407.5210, July 2014.

[14] P. Giselsson and S. Boyd, "Linear convergence and metric selection for DouglasRachford splitting and ADMM," IEEE Transactions on Automatic Control, vol. 62, pp. 532-544, Feb 2017.

[15] P. Giselsson, "Tight global linear convergence rate bounds for DouglasRachford splitting," Journal of Fixed Point Theory and Applications, 2017. doi:10.1007/s11784-017-0417-1.

[16] F. Deutsch, The Angle Between Subspaces of a Hilbert Space, pp. 107-130. Dordrecht: Springer Netherlands, 1995.

[17] H. H. Bauschke, J. Y. B. Cruz, T. T. A. Nghia, H. M. Pha, and X. Wang, "The rate of linear convergence of the Douglas-Rachford algorithm for subspaces is the cosine of the Friedrichs angle," Journal of Approximation Theory, vol. 185, no. 0, pp. 63-79, 2014.

[18] H. H. Bauschke, J. Y. B. Cruz, T. T. A. Nghia, H. M. Pha, and X. Wang, "Optimal rates of linear convergence of relaxed alternating projections and generalized Douglas-Rachford methods for two subspaces," Numerical Algorithms, vol. 73, no. 1, pp. 33-76, 2016.

[19] M. Grant and S. Boyd, "CVX: Matlab software for disciplined convex programming, version 3.0." http://cvxr.com/cvx, 2016. 
[20] S. Diamond and S. Boyd, "CVXPY: A Python-embedded modeling language for convex optimization," Journal of Machine Learning Research, vol. 17, no. 83, pp. 1-5, 2016.

[21] M. Udell, K. Mohan, D. Zeng, J. Hong, S. Diamond, and S. Boyd, "Convex optimization in Julia," SC14 Workshop on High Performance Technical Computing in Dynamic Languages, 2014.

[22] B. O’Donoghue, E. Chu, N. Parikh, and S. Boyd, "Conic optimization via operator splitting and homogeneous self-dual embedding," Journal of Optimization Theory and Applications, vol. 169, no. 3, pp. 1042-1068, 2016.

[23] J. Liang, J. Fadili, G. Peyré, and R. Luke, Activity Identification and Local Linear Convergence of Douglas-Rachford/ADMM under Partial Smoothness, pp. 642653. Cham: Springer International Publishing, 2015.

[24] L. Demanet and X. Zhang, "Eventual linear convergence of the Douglas-Rachford iteration for basis pursuit," Mathematics of Computation, vol. 85, no. 297, pp. 209-238, 2016.

[25] M. Fält and P. Giselsson, "Line search for generalized alternating projections," in 2017 American Control Conference (ACC), 2017.

[26] J. Bezanson, A. Edelman, S. Karpinski, and V. B. Shah, "Julia: A fresh approach to numerical computing," SIAM Review, vol. 59, no. 1, pp. 65-98, 2017. 